\title{
DEVELOPMENT OF A FreE-Flight SiMUlation InFRASTRUCTURE
}

\author{
Eric S. Miles \\ Paul C. Davis \\ Seagull Technology, Inc. \\ 16400 Lark Ave., Los Gatos, CA 95032 \\ David J. Wing \\ NASA Langley Research Center \\ Hampton, VA 23681
}

\begin{abstract}
In anticipation of a projected rise in demand for air transportation, NASA and the FAA are researching new air-traffic-management (ATM) concepts that fall under the paradigm known broadly as "free flight". This paper documents the software development and engineering efforts in progress by Seagull Technology, to develop a free-flight simulation (FFSIM) that is intended to help NASA researchers test mature-state concepts for free flight, otherwise referred to in this paper as distributed air / ground traffic management (DAG TM). Under development is a distributed, humanin-the-loop simulation tool that is comprehensive in its consideration of current and envisioned communication, navigation and surveillance (CNS) components, and will allow evaluation of critical air and ground traffic management technologies from an overall systems perspective. The FFSIM infrastructure is designed to incorporate all three major components of the ATM triad: aircraft flight decks, air traffic control (ATC), and (eventually) airline operational control (AOC) centers.
\end{abstract}

\section{INTRODUCTION}

Over the past twenty years, increased demand for air travel has outpaced the design of the National Airspace System (NAS). Insufficient capacity, limited access, and excessive operating restrictions have led to significant increases in user costs and delays and an overall decrease in efficiency for all users ${ }^{1}$. Furthermore, the market demand for air travel is expected to increase several-fold in the coming decade. Recent technological advances now provide the opportunity to redesign the NAS to substantially increase its capacity to accommodate air traffic growth and to provide substantial operational flexibility to airspace users, while maintaining, and possibly improving, safety.

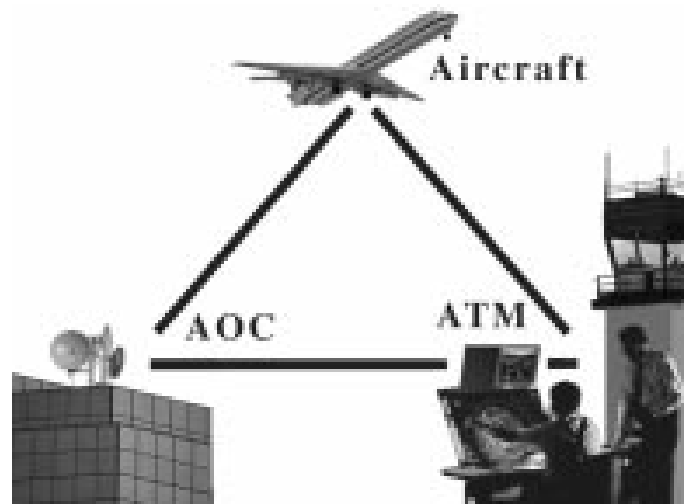

Figure 1: The FFSIM infrastructure is designed to incorporate all three major components of the ATM triad: aircraft flight decks, air traffic control (ATC), and airline operational control (AOC) centers.

A broad concept of NAS operations known as "free flight", proposed by the RTCA ${ }^{1}$ in 1995 , may provide these and other benefits through application of new technologies and procedures. The primary difference between today's directroute-clearance approach and free flight will be the pilot's ability to operate the flight without the requirement to follow specific route, speed, and altitude clearances. In mature visions of free flight, the flight crew of properly equipped aircraft would be given the authority to maneuver at will while broadcasting their intentions to other system participants and maintaining responsibility for ensuring minimum separation standards are met. Aircraft without the equipment necessary for performing these functions would receive clearances from the ground through voice or data

Copyright $\odot 1999$ by The American Institute of Aeronautics and Astronautics, Inc.

No copyright is asserted in the United States under Title 17, U.S. Code. The U.S.

Government has a royalty-free license to exercise all rights under the copyright

claimed herein for Governmental purposes. All other rights are reserved by the

copyright owner. 
link communications, and separation assurance responsibility for these aircraft would remain with the ground-based air traffic service provider (ATSP). This distribution of responsibility and authority will be referred to in this paper as distributed air / ground traffic management (DAG TM).

In recent years, the ATM community has been focused on the development of technologies and evolutionary improvements in efficiency and capacity based on the current mode of groundbased air traffic control. The Center-TRACON Automation System (CTAS) under development by NASA ${ }^{2,3}$ will provide air traffic controllers a set of tools to better manage scheduling and sequencing of aircraft into the terminal area with continuous updating to the runway threshold. Limited implementation of CTAS and other tools is underway through the FAA's Free Flight Phase I program. Isolated demonstrations and evaluations of other airborne technologies related to the advancement of ATM are underway in the FAA's Safe Flight 21 project.

In contrast to the development of individual airborne and ATM technologies, less attention has been given to date to establishing the overall system feasibility of mature-state free-flight concepts and the integration of essential underlying CNS components. The work documented in this paper leverages these past and existing airborne and ATM research endeavors while filling a void in the ATM research community by providing a mechanism for evaluating the system-level feasibility of free-flight operations and the integration of enabling technologies, including CNS components and decision support tools (DSTs), for both the flight crew and the air traffic service providers on the ground.

\section{RELATED DEVELOPMENTS}

Several recent and current development efforts are relevant to the FFSIM infrastructure development. The efforts are briefly described below, and the connection of each to FFSIM development or NASA research is identified. A thorough discussion of these endeavors is beyond the scope of this paper.

Ground ATM Decision Support Tools

NASA researchers have been developing and fieldtesting CTAS, a DST for ground-based air-traffic management ${ }^{2,3}$. Core components of CTAS are leveraged in the FFSIM infrastructure to provide the situation display for the ATSP station. (The notion in free flight of distributing traffic management functions between the ground and the air leads to the re-designation of air traffic controller to air traffic service provider.) Inclusion of these CTAS core components allows for eventual expansion to include CTAS components such as the Traffic Management Advisor (TMA) for scheduling and En-route / Descent Advisor (E/DA) for ATSP decision support.

\section{Flight Simulation Tools}

NASA has developed a versatile PC-based simulation of a transport-category aircraft called "FastWin" (FMS-Autoflight Simulation Tools for Windows) ${ }^{4}$. FastWin includes an aircraft performance model, a Flight Management System (FMS) model, Control Display Unit (CDU), Mode Control Panel (MCP), and primary cockpit displays. FastWin is integrated into the FFSIM infrastructure as the core component of the research pilot station.

Logicon previously developed a software simulation tool entitled "Pseudo Aircraft Systems" (PAS) to serve as a target generation facility and pseudo-pilot station with a low-fidelity auto-pilot capability for CTAS development experiments 5 . The PAS software is used in the FFSIM infrastructure to manage traffic scenarios, to generate targets, and to provide the ability for a human operator to control multiple pseudo-pilots.

The National Aerospace Laboratory of The Netherlands (NLR) is currently conducting extensive ATM research activities through humanin-the-loop simulation. Notable simulation tools developed by NLR include TMX, a target-aircraft generator; AIRSIM, an interface to a single pilot station; and NARSIM, a distributed simulation effort aimed at investigating the European version of free flight ${ }^{6}$. A close working relationship has been established between Seagull and NLR to exploit lessons learned during their research and to accelerate FFSIM design and development. NASA and NLR are exploring a collaborative approach to free-flight research with activities starting in 1999.

Eurocontrol has also been very active in ATM research. Efforts relevant to FFSIM include: OASIS, a generic simulation infrastructure based on the Common Object Request Broker Architecture (CORBA); ESCAPE, a distributed air traffic control (ATC) simulation that uses OASIS; and FREER, a four-part research program that is 


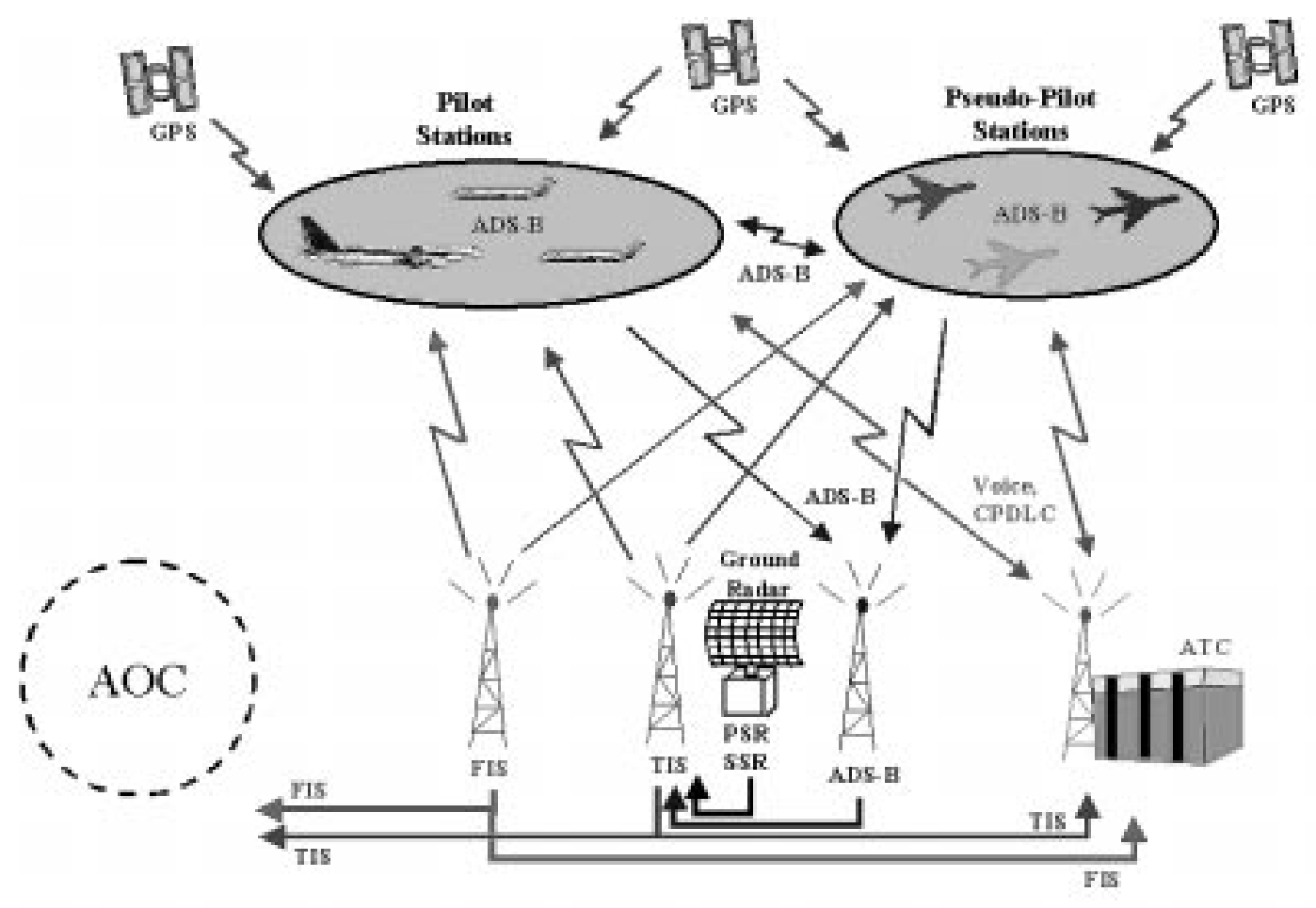

Figure 2: The FFSIM infrastructure is a distributed simulation tool capable of providing a comprehensive model of current and envisioned air-traffic-management operations with future communication, navigation, and surveillance capabilities.

simulating, developing, and flight-testing a prototype system for fully-autonomous aircraft operations.

\section{Conflict Detection and Resolution}

Numerous organizations have developed software algorithms for conflict detection and resolution $(\mathrm{CD} \& \mathrm{R})^{7}$. Seagull Technology has developed a solution approach that can be applied in both ground and airborne contexts ${ }^{8}$. MIT has performed significant research in $\mathrm{CD} \& \mathrm{R} 9{ }^{9}{ }^{10}$. Lincoln Laboratory at MIT developed a self-organizational approach for conflict resolution ${ }^{11}$. This last approach, combined with conflict detection based on aircraft state information and flight plans, was implemented as an initial decision-aiding prototype system in FFSIM for the flight deck and the ATSP by Lockheed Martin Aeronautical Systems ${ }^{12}$.

\section{CNS Modeling}

MIT Lincoln Labs has extensively studied the automatic dependent surveillance - broadcast (ADS-B) concept ${ }^{13}, 14$. These theoretical and empirical studies of ADS-B, which include message-collision modeling, are leveraged in the FFSIM representation of the CNS infrastructure. NASA Glenn Research Center is currently developing high-fidelity parametric models for communication mechanisms such as Mode $\mathrm{S}$, VHF Datalink (VDL) Modes 2, 3, and 4, etc. Many of these models will be incorporated into FFSIM to permit assessment of design requirements for freeflight application of these technologies.

\section{FFSIM INFRASTRUCTURE AND RESEARCH \\ CAPABILITIES}

The FFSIM infrastructure is unique in that it provides system-level integration and modeling of technologies vital for free flight with sufficient fidelity for assessing their impact on the feasibility of free-flight operations. FFSIM provides a humanin-the-loop environment where several researchsubject pilots fly simulated aircraft through simulated airspace managed by one or more research-subject ATSPs. The human-subject pilots and ATSPs may have the assistance of prototype DSTs providing separation assurance and other navigation functions and may communicate by voice or digital communications. CNS technologies are modeled with moderate realism to allow the processes of communication, navigation, and surveillance to occur within the free-flight environment with realistic characteristics and limitations. Additionally, the simulated airspace may be populated by target aircraft controlled by 
human participants responding to voice clearances issued by the ATSPs.

FFSIM will provide research capabilities in several areas. First, it will allow the assessment of CNS infrastructure design characteristics with respect to free-flight operations. Performance standards are emerging for technologies vital to free flight without the benefit of requirements generated under free-flight operating conditions. Data generated by FFSIM will begin to shed light on these requirements. Second, FFSIM will provide a means for assessing human performance and procedures to the extent appropriate in a mediumfidelity simulation environment. The type and presentation of information required by the human participants to maintain awareness and to perform the necessary duties of their stations can be assessed. Third, parametric controls on important factors such as wind-prediction accuracy will allow determination of trajectory-prediction requirements for conflict detection. Fourth, FFSIM will permit assessment of CD\&R feasibility in both level and transition phases of flight and in a variety of airspace-constrained and schedule-constrained scenarios. In particular, the ability of aircraft transitioning to terminal-area operations to maintain separation while adhering to operational air traffic scheduling is critical for assessing feasibility of free flight since the vast majority of U.S. domestic airspace contains aircraft in this mode of operation. Additionally, this ability must be assessed in the presence of aircraft with mixed levels of equipage (and therefore varying ability to self-separate).

The current scope of FFSIM development focuses on the ATSP and aircraft flight deck aspects of DAG TM operations. However, the FFSIM infrastructure is designed to facilitate the incorporation of a future AOC presence, as shown in Figure 1. The FFSIM infrastructure allows participants to emulate the roles of pilots and pseudo-pilots, ground-based controllers, and, eventually, airline dispatchers.

\section{FFSIM INFRASTRUCTURE COMPONENTS}

The FFSIM infrastructure is a distributed simulation tool comprised of many components. The majority of these components are depicted in Figure 2. Development emphasis to date has focused on the aircraft flight deck, the ATSP, and the CNS infrastructure components. Modeling of the AOC and information sharing between the AOC and the flight deck and in the ATSP are planned for future phases of FFSIM development. A brief description of each FFSIM component follows.

\section{$\underline{\text { Pilot Stations }}$}

The airborne portion of the FFSIM infrastructure is comprised of two types of flight-deck environments. The first environment, the "pilot station", provides an opportunity for several humans (i.e. research-subject pilots) to each control a single aircraft in real time with a reasonably high level of interface fidelity. The pilot station is implemented on one or two PC workstations and is comprised of an aircraft performance model, an interactive flight management system (FMS), a primary-flight display (PFD), a navigation display (ND), a mode control panel (MCP), communications, navigation, and surveillance equipment. These components, with the exception of the CNS equipment, are represented by the NASA-developed FastWin software package, which has been integrated with the FFSIM infrastructure. Figure 3 depicts the FastWin PFD and ND used for the FFSIM pilot station. The navigation display will be evolved into an integrated multifunction display (MFD) that will include navigation, flight plan, traffic, weather, and other data, in a user-configurable, layered format.

The CNS equipment models the hardware components required by the pilot station. Components include a voice transceiver for twoway verbal communication with the ATSP (and eventually the AOC), a transceiver for two-way digital controller-pilot data link communications (CPDLC) with the ATSP (and eventually the AOC), a global positioning system (GPS) receiver, an ADS-B transceiver, and receivers for Traffic Information Services (TIS) data and Flight Information Services (FIS) data.

The pilot stations may be configured to contain an integrated DST that accepts information regarding its own airplane, other traffic, and NAS status. The FFSIM infrastructure allows an integrated DST to output recommended conflict-free flight trajectories that adhere to specified constraints. The development of such DSTs is not within the scope of the FFSIM infrastructure development. 


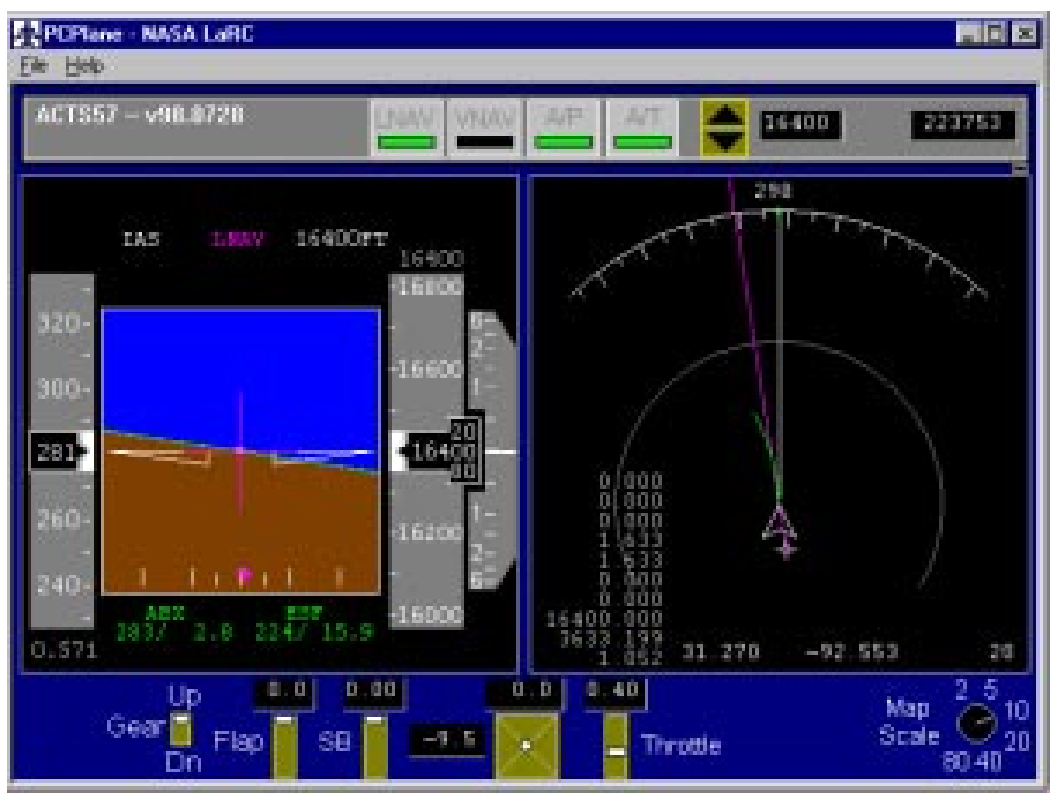

Figure 3: The FastWin PFD and Navigation Displays are integral parts of the FFSIM research pilot station. (Courtesy of NASA)

\section{Pseudo-Pilot Stations}

The second flight-deck environment in the FFSIM infrastructure is the pseudo-pilot station, which allows several humans (i.e. pseudo pilots) to each control multiple aircraft (up to a dozen each) in real time with a low level of interface fidelity. The pseudo-pilot capability enables FFSIM traffic scenarios to be populated with many aircraft operated by just a few humans.

Similar to the pilot station, the pseudo-pilot station contains an auto-pilot capability, CNS equipment, and possible instances of DSTs similar to those that would serve the research pilot stations. No individual cockpit displays such as the PFD and MFD are included in the pseudo-pilot station. The pseudo-pilot station auto-pilot and control interface are represented by the PAS software. The pseudopilot CNS equipment is essentially the duplicate of that employed by the pilot stations and is configured to serve multiple aircraft.

\section{Air Traffic Service Provider Stations}

The FFSIM ground infrastructure includes an ATSP component that allows human subjects to regulate and separate air traffic according to both current and future (envisioned) operating procedures for ATM. The central ingredient of the FFSIM ATSP component is the NASA-developed CTAS software. Figure 4 depicts the CTAS horizontal situation display known as the "pGUI". Multiple ATSP stations representing adjacent sectors can operate simultaneously, allowing research to pursue multi-sector coordination issues.

The FFSIM infrastructure provides both voice and digital communications between the ATSP and all appropriately equipped aircraft allowing for simulation of both current and various future concepts for air / ground interaction.

The ATSP component of the FFSIM infrastructure is designed to incorporate ground-based DSTs. However, the development of such DSTs is not within the scope of the FFSIM infrastructure development.

\section{Communication, Navigation and Surveillance}

\section{Components}

The comprehensive modeling of CNS components at a level of fidelity sufficient for concept feasibility assessment and operational requirements definition is a major focus of this development effort. The ability to selectively increase the fidelity of any component has been designed into the FFSIM infrastructure. Each of the CNS infrastructure components is briefly described below.

\section{Automatic Dependent Surveillance -}

\section{Broadcast}

ADS-B messages are broadcast by all aircraft equipped with an ADS-B transceiver and received by all similarly-equipped aircraft and the ATSP. 
Both the broadcast rate and the nominal operating range for ADS-B are scenario parameters that can be configured by the researcher.

Each ADS-B message will contain, at a minimum, the following basic message content: computer identification number, timestamp, aircraft type, position source, latitude, longitude, altitude source, altitude, ground speed, ground-track direction, and vertical speed. The level of intent (i.e., the number of intended future trajectory change points) to be included in the ADS-B message can be configured by the researcher.

The FFSIM infrastructure modeling of ADS-B will be initially consistent with Mode-S data communication, yet will not preclude the option of future modeling of other viable technologies capable of implementing ADS-B.

\section{Ground-Based Surveillance}

Radar is the centerpiece surveillance system that enables current air traffic control. It is projected to continue to play a vital, albeit diminished, role as operations evolve toward free flight. The FFSIM infrastructure modeling of ground-based surveillance will include both primary and secondary surveillance radar (PSR and SSR).

The PSR model will include the Air Route Surveillance Radar (ARSR), used in the en-route airspace, and the Airport Surveillance Radar (ASR), used in the TRACON airspace ${ }^{15}$. The SSR model will include the interrogation-reply interaction process of secondary surveillance, including the envisioned ground-initiated communication broadcast (GICB) concept detailed in ${ }^{16}$. In the GICB concept, the transponder reply to ground interrogation will include the most recent ADS-B message of the interrogated aircraft, permitting independent verification of ADS-B messaging on the ground.

\section{Traffic Information Services}

As depicted in Figure 2, TIS provides a compilation of ground-received ADS-B message data and PSR/SSR data that is broadcast to all equipped aircraft, the ATSP, and the AOCs. The TIS concept allows aircraft that are equipped with a TIS receiver, but not with an ADS-B transceiver,

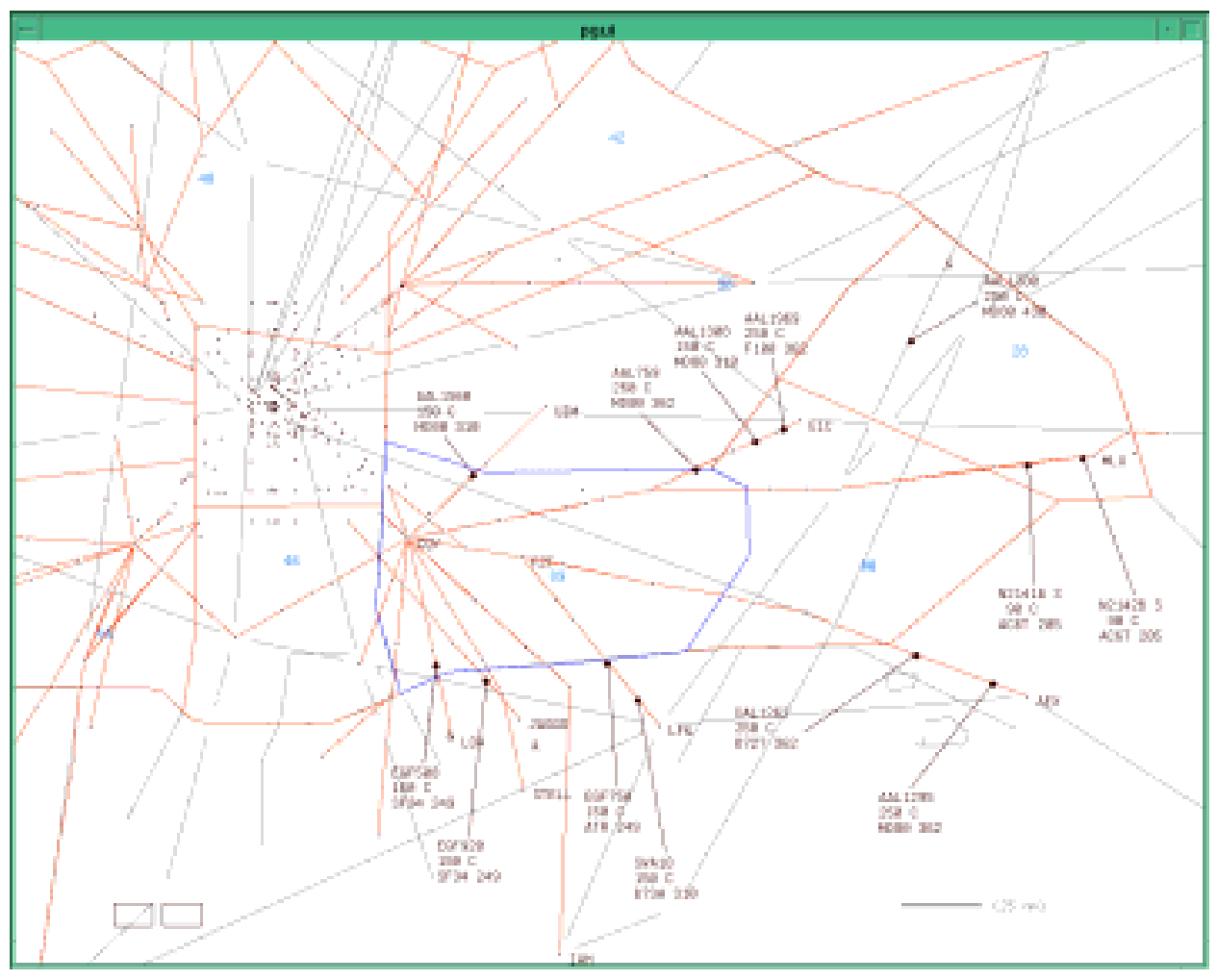

Figure 4: The CTAS pGUI controller display, shown here for ZFW, comprises the main controller display for the FFSIM infrastructure. (Courtesy of NASA) 
to receive the position, velocity and intent content of neighboring aircraft ${ }^{17}$. For aircraft equipped with ADS-B, TIS provides a redundant source of traffic information. The TIS broadcast rate and nominal-operating range are scenario parameters that can be configured by the researcher.

\section{Flight Information Services}

FIS is a broadcast of weather, wind, special-use airspace (SUA), and other pertinent information from the ground to equipped aircraft ${ }^{18}$. A variety of FIS sources, such as NEXRAD weather radar, METARs, etc., will provide the FIS data content.

The FFSIM infrastructure modeling of FIS will be initially consistent with VDL Mode-2 technology, a likely candidate for FIS realization. Again, other viable technologies capable of implementing FIS will not be precluded. The FIS broadcast rate and nominal-operating range are scenario parameters that can be selected by the researcher.

Controller-Pilot Data Link Communications CPDLC allows for digital communications between the ATSP station and the pilot and pseudo-pilot stations. A graphical interface will be implemented for both the pilot and ATSP stations to facilitate the use of CPDLC ${ }^{19}$.

CPDLC is envisioned for many applications in current and future ATM operations, including the up-link of clearances, route deviations, and new trajectories from the ATSP to the pilot. One application of CPDLC, important in the DAG TM concept, is the digital up-link of conflict resolution maneuvers from a ground-based DST to the pertinent aircraft. Pilots will use CPDLC to respond to and possibly request ATSP instructions, as well as inform ATSP of changes to the current flight trajectory.

The FFSIM infrastructure modeling of CPDLC will be initially consistent with VDL Mode 3 technology, a likely candidate for realizing CPDLC. Other communication technologies for implementing CPDLC will not be precluded.

\section{Global Positioning System Navigation}

The position and velocity data contained in each ADS-B message is provided by a GPS satellite navigation receiver model. The FFSIM infrastructure includes a stochastic error model of selective availability (SA) for the basic navigation signal $^{20,21}$.
Modeling of wide-area and local-area augmentation system (WAAS and LAAS) accuracy levels are planned as future enhancements.

\section{Airline Operational Control}

The third and final entity of the ATM operations triad depicted in the lower left of Figure 1 is the AOC facility. Although the initial development effort has focused on the flight deck and ATSP aspects of ATM operations, the FFSIM infrastructure is designed to facilitate incorporation of an AOC presence in the future, complete with all the communication channels between the AOC, the flight decks, and the ATSP.

\section{SOFTWARE IMPLEMENTATION}

This section describes details of the FFSIM infrastructure software.

\section{CORBA}

The FFSIM infrastructure employs the Common Object Request Broker Architecture (CORBA) ${ }^{22}$. CORBA is a software industry standard for facilitating communication between objects in distributed applications. An important aspect of CORBA is the interface definition language (IDL). The IDL allows language independent software interfaces to be specified. An interface specified in IDL is compiled into code stubs for a specified target language, e.g. C++ or Java. A programmer creating a CORBA server must implement the skeleton methods in the generated code stubs. To create a client to the server, a programmer simply needs to create a proxy to the server by using the generated code.

CORBA was chosen over a more traditional TCP/IP approach because of its languageindependent, object-oriented, and standardized nature. It was also felt, and later substantiated, that CORBA would be a faster and less error prone method for developing inter-process communications. Eurocontrol's successful implementation of CORBA for ATM simulations also contributed to the decision.

\section{CORBA Services}

The CORBA specification outlines standard services that are intended to provide generic support to any CORBA system. FFSIM is designed to leverage some of these services.

The CORBA Naming Service is used to store object names in a repository. Conceptually it is similar to the white pages of a telephone directory. 
FFSIM uses the Naming Service to store names of objects that represent individual aircraft components as well as the names of simulation services. The object names exist in the Naming Service repository even when the simulation is not active. This allows a client to search the Naming Service and retrieve a specific object. If the server that contains that object is inactive the object will be automatically launched.

The CORBA Event Service is used to de-couple the clients from the servers. This service allows servers to publish specific types of events and clients to subscribe to those events. The server supplying the event does not need knowledge of the client consuming the event. A new service called the Notification Service extends the functionality of the Event Service and provides "quality of service" delivery of events. While currently not used in FFSIM, the Event Service or its replacement the Notification Service, will be a critical part of the future infrastructure.

The Time Service is another CORBA service that may be incorporated into FFSIM. The Time Service provides distributed time management for a CORBA system. Unlike the Naming, Event, and Notification services a commercial version of the Time Service is not available. Any Time Service created for FFSIM will also need to be extended to include simulation time management.

\section{Component Integration}

The CTAS, PAS, and FastWin software all handle external communication using TCP/IP sockets. These applications are representative of a large number of existing components that do not use CORBA, but may require integration into FFSIM. There are two approaches for integrating existing TCP/IP components into FFSIM.

The first approach is to add a CORBA interface to the existing component. The advantage of this approach is that the application will be completely "plugged" into the simulation and will be able to easily take advantage of any services provided by other CORBA components. The disadvantage of this approach is that it may require extensive modifications, depending on the organization of the original source code.

The second approach is to create a new process that can communicate with the non-CORBA application using an existing TCP/IP interface and with the rest of the simulation using CORBA. The advantage of this approach is that, depending on the state of the existing application, little if any source code modifications are required. This may also be the only option if source code cannot be modified. The disadvantage of this approach is that it adds a layer of overhead and can be more time consuming to implement than directly integrating CORBA.

The FFSIM infrastructure uses the second approach for integrating FastWin. The FastWin application contains three separate processes that would potentially need to be modified to integrate with CORBA. Because FastWin is a research tool, NASA is continuously updating it. For these reasons it was decided that the best approach was to create a single CORBA to TCP/IP bridge and minimize changes to the existing FastWin code.

The integration of PAS into FFSIM was also accomplished using a CORBA to TCP/IP bridge. The primary reason for using a bridge in this instance was the proprietary nature of the PAS source code prevented direct modifications.

\section{Interfaces}

The FFSIM infrastructure interfaces were created using the CORBA IDL. The IDL provides greater flexibility than a traditional byte or text message sent over a TCP/IP socket. The CORBA interfaces create a simulation databus. Figure 5 illustrates the CORBA communication between components within the pilot station. Using the existing FFSIM interfaces a new component could easily be added.

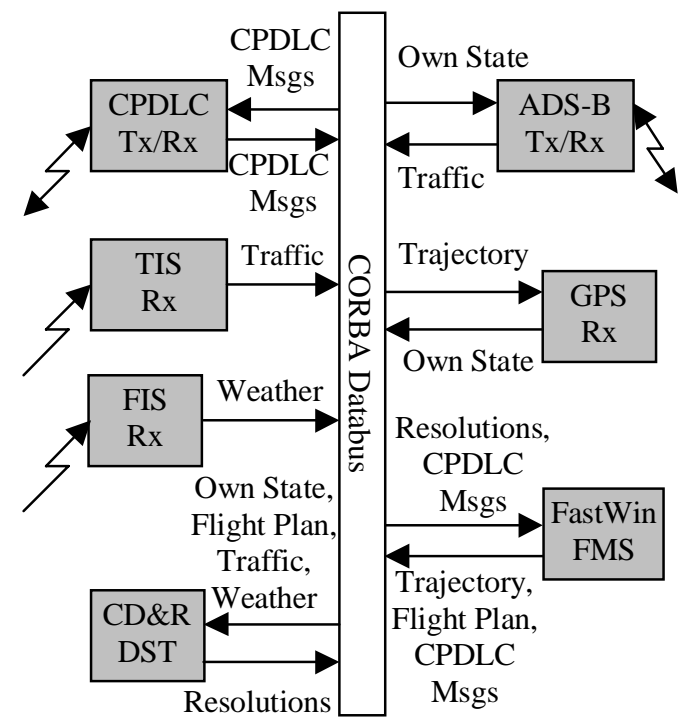

\section{Figure 5: Pilot Station Component Communication.}




\section{Message Filtering}

In some cases the FFSIM interfaces provide access to more data than is realistic or even necessary. However, this makes it easier to examine future concepts where this additional data may be needed.

One strategy the FFSIM infrastructure uses is client-side filtering. For example, an airborne CPDLC transceiver broadcasts and receives datalink messages to and from an ATSP CPDLC transceiver on the ground. The probability of a successful transmission is determined by many factors including the distance between transceivers. The receiving CPDLC transceiver determines if a message is successfully received based on the distance to the transmitting transceiver. In order to accomplish this the receiver must know information about the transmitter that may not be contained in the message, e.g. its location. The solution is to provide this information in a message header that is only used by the CPDLC transceiver and not passed along to any other ground or airborne applications. It is left to the individual implementation to decide what to do with the information in the message header. In the case of the CPDLC transceiver, the message receiver would use the location of the transmitter to determine if the transmitter is out of range. If it does determines that the transmitter is out of range the message is logged as being dropped and then ignored as if it was never received. The concept of client-side message filtering allows the component developer to decide what information should be provided to the component.

\section{Deployment}

FFSIM is a distributed multi-platform workstation based simulation that currently runs on computers with Solaris and Windows NT operating systems. The nominal FFSIM configuration consists of three pilot stations, three pseudo-pilot stations, two controller stations, and a simulation server station.

The CTAS ATSP software and the PAS pseudopilot software run on Solaris. The FastWin software, the CNS equipment, and the simulation infrastructure run on Windows NT.

\section{CONCLUSIONS}

A medium-fidelity workstation-based simulation of airspace operations is under development and has reached its first phase of completion. The Free Flight Simulation (FFSIM) was designed specifically to permit exploration of a proposed future mode of operations known as "free flight". In mature visions of free-flight operations, both the authority to approve changes in trajectory and the responsibility to ensure regulatory separation from other aircraft are distributed between aircraft flight crews and ground-based air traffic service providers.

The FFSIM infrastructure provides a simulated airspace environment complete with communication, navigation, and surveillance component models. Human operators participate in the simulation by controlling aircraft as pilots or pseudo pilots, managing airspace as air traffic service providers, and interacting with the other human operators in the simulation while using simulated technology appropriate for their role in free-flight operations.

The simulation was designed as a research tool to permit investigation of a variety of research issues and the development of technology requirements for free-flight operations. Examples of research areas appropriate for investigation in FFSIM include human actions and interactions in a distributed air/ground traffic management environment, procedures for free-flight operations, time horizons, feasibility of aircraft to autonomously maintain regulatory separation while efficiently meeting scheduled arrival constraints, and many other human-factors and engineeringrelated research issues. Examples of technology design appropriate for FFSIM usage include determining requirements for surveillance range and latency, intent broadcast and inference, wind and trajectory prediction accuracy, conflict detection and resolution algorithms, and essentially any variable under parameter control in FFSIM.

Much progress has been made in improving capacity and efficiency in the current mode of NAS operations through efforts such as CTAS. Free flight offers the possibility for significant increases in the ability of the NAS to accommodate air traffic growth and significant increases in operational flexibility for airspace users, while maintaining and possibly improving safety. A simulation capability, FFSIM, has now been developed that will serve as a research tool in defining technology requirements and determining feasibility of the free-flight operational concept.

\section{REFERENCES}

[1] Report of the RTCA Board of Directors' Select Committee on Free Flight, RTCA, January 1995.

[2] Erzberger, H., Davis, T. J., and Green, S., "Design of Center-TRACON Automation System," 
AGARD Guidance and Control Symposium on Machine Intellignce in Air Traffic Management, Berlin, Germany, May 1993.

[3] Denery, D. G. and Erzberger, H., "The CenterTRACON Automation System: Simulation and Field Testing," Proceedings of the advanced Workshop on ATM (ATM 95), sponsored by the National Research Council of Italy, Capri, Italy, Oct. 2-6, 1995. (Also published as NASA Technical Memorandum 110366, August 1995).

[4] Palmer, M. T., Abbott, T. A., Williams, D. H., "Development of Workstation-Based Flight Management Simulation Capabilities within NASA Langley's Flight Dynamics and Control Division", Ninth International Symposium on Aviation Psychology, Columbus, OH, April, 28-May, 1, 1997.

[5] Pseudo-Aircraft Systems (PAS) Users's Manual, Revision 4.2, Logicon/Syscon/Syre, November 1997.

[6] NLR Web Site, www.nlr.nl, and personal discussions.

[7] Kuchar, J. K. and L. C. Yang, "Survey of Conflict Detection and Resolution Modeling Methods", AIAA-97-3732, AIAA Guidance, Navigation, and Control Conference, New Orleans, LA, August 11-13, 1997.

[8] Krozel, J. and Peters, M. "Conflict Detection and Resolution for Free Flight", Air Traffic Control Quarterly, Vol. 5, No. 3, pp. 181-212, 1997.

[9] Yang, L. C., and J. K. Kuchar, "Prototype Conflict Alerting Logic for Free Flight", AIAA Journal of Guidance, Control, and Dynamics, Vol. 20, No. 4, July-August 1997.

[10] Kuchar, J. K. and L. C. Yang, "Incorporation of Uncertain Intent Information in Conflict Detection and Resolution", 36th IEEE Conference on Decision and Control, San Diego, CA, December 10-12, 1997.
[11] Eby, Martin S., "A Self-Organizational Approach for Resolving Air Traffic Conflicts", The Lincoln Laboratory Journal, Vol. 7, No. 2, 1994.

[12] "User Instruction Manual for NASA Research Task Order (RTO) -5(A) Free Flight Simulation Components Development", Lockheed Martin Corporation, January 1999.

[13] Orlando, V. A., Harmon, W. H., GPS-Squitter Capacity Analysis, MIT Lincoln Laboratory, Project Report ATC-214, 1994.

[14] Minimum Aviation System Performance Standards for Automatic Dependent Surveillance Broadcast (ADS-B), RTCA, SC-186, DO-242, 1998.

[15] M. S. Nolan, Fundamentals of Air Traffic Control, $2^{\text {nd }}$ Ed., Wadsworth, Inc., 1994.

[16] National Airspace System ArchitectureVersion 4.0, Federal Aviation Administration, ASD-1, January 1999.

[17] Minimum Operational Performance Standards for Traffic Information Service (TIS) Data Link Communications, RTCA, SC-169, DO-239, 1997.

[18] Operational Concepts for Data Link Applications of Flight Information Services (FIS), RTCA, SC-169, DO-232, 1996.

[19] Minimum Operational Performance Standards for ATC Two-Way Data Link Communications, RTCA, SC-169, DO-219, 1993.

[20] Global Positioning System: Theory and Applications, Vol. 1, AIAA, Vol.163, pp 606-608, 1996.

[21] "Global Positioning System Standard Positioning Service Signal Specification," $2^{\text {nd }}$ Ed., DoD GPS/JPO, p. B-18, June 2, 1995.

[22] OMG Web Site, www.omg.org. 